\title{
Perturbative renormalization in parton distribution functions using Overlap fermions and Symanzik improved gluons
}

\author{
M. Ioannou and H. Panagopoulos \\ Department of Physics, University of Cyprus, Nicosia CY-1678, Cyprus \\ e-mail: mike80bi@yahoo.gr, haris@ucy.ac.cy
}

\begin{abstract}
We calculate the 1-loop renormalization of the fermion self-energy, all local fermion bilinears, as well as a set of extended bilinears which form a basis corresponding to moments of the parton distribution functions.

We use the overlap action for fermions and Symanzik improved action for gluons.

Our results are presented as a function of the overlap parameter $\rho$ and the parameters entering the Symanzik action.
\end{abstract}




\section{Introduction.}

In recent years, simulations in lattice QCD have employed an ever increasing number of "improved" actions for gluon and fermion fields. This practice is well motivated, since it leads to a reduction of $\mathcal{O}(a)$ effects ( $a$ : lattice size), thus resulting in a faster approach to continuum physics. In the case of fermions, certain improved actions carry the crucial added advantage of preserving chiral symmetry on the lattice.

The variety of lattice actions presently in use calls for the computation of a large number of renormalization functions, before a comparison to physical results can be attempted; such functions often pertain to fermion composite operators, whose matrix elements are widely used to extract mass spectra, decay rates and transition amplitudes.

A set of operators which are of particular interest are fermion bilinears; they can be classified according to the representations of the rotation group, and the global flavor group. Operators of this kind appear naturally in the operator product expansion (OPE), as applied to hadronic physics; in the Bjorken limit, involved in studies of deep inelastic scattering, the OPE for a product of hadronic currents takes the form:

$$
J(x) J(0) \sim \sum_{n, i} C^{n, i}\left(x^{2}\right) x^{\mu_{1}} \ldots x^{\mu_{n}} O_{\mu_{1} \ldots \mu_{n}}^{(n, i)}(0)
$$

The forward matrix elements of the local operators $O^{(n, i)}$ appearing in this expansion are directly related to the moments of hadron structure functions. The dominant contribution in the expansion is given by operators whose twist (dimension minus spin) equals two, which in the flavor non-singlet case means the symmetric traceless operators [1]

$$
\begin{gathered}
O_{\mu \mu_{1} \ldots \mu_{n}}=\bar{\psi} \gamma_{\{\mu} D_{\mu_{1}} \ldots D_{\left.\mu_{n}\right\}} \frac{\lambda^{a}}{2} \psi \\
O_{\mu \mu_{1} \ldots \mu_{n}}^{(5)}=\bar{\psi} \gamma_{\{\mu} \gamma_{5} D_{\mu_{1} \ldots D_{\left.\mu_{n}\right\}}} \frac{\lambda^{a}}{2} \psi
\end{gathered}
$$

where $\lambda^{a}$ are flavor matrices, and the curly brackets denote symmetrization over Lorentz indices. A proper renormalization is required for these operators, before one can relate their matrix elements, as extracted from numerical simulations, to physically observable quantities.

In this work we employ Symanzik improved actions for gluons. We consider, in particular, actions made up of closed Wilson loops with up to 6 links; they are the most widely used cases in numerical simulations. This class of actions is described by 3 independent parameters which are tuned either in perturbation theory (at tree level or beyond) or through simulation, so as to minimize $\mathcal{O}(a)$ effects. Particular choices of action within this class, aside from the standard Wilson action, are: tree level Symanzik improved, "tadpole improved" Lüscher-Weisz, Iwasaki and DBW2.

For the fermion part of the action we employ Neuberger's overlap-Dirac operator. This action is characterized by one free parameter. The definition of this operator involves the inverse square root of $X^{\dagger} X$, where $X$ is the Wilson-Dirac operator with a negative mass term; this operation is extremely costly in numerical simulations, and its viability depends 
critically on the condition number of $X^{\dagger} X$. Thus, an improved action for gluons becomes all the more important, since it tends to decrease the condition number [2].

The quantities whose renormalizations we set out to compute, to 1-loop order in lattice perturbation theory, are:

a) The quark propagator

$$
\left\langle\psi_{\alpha}(x) \bar{\psi}_{\beta}(y)\right\rangle
$$

b) Local fermion bilinears.

$$
O_{X}=\bar{\psi}(x) \Gamma^{X} \psi(x)
$$

c) Operators which measure the first moment of quark momentum distributions.

$$
\begin{gathered}
O_{V_{2}}=\bar{\psi} \gamma_{\{1} D_{4\}} \psi \\
O_{V_{2}}^{\prime}=\bar{\psi} \gamma_{4} D_{4} \psi-\frac{1}{3} \sum_{i=1}^{3} \bar{\psi} \gamma_{i} D_{i} \psi
\end{gathered}
$$

d) Operators which measure the first moment of quark helicity distributions.

$$
\begin{gathered}
O_{A_{2}}=\bar{\psi} \gamma_{\{1} \gamma_{5} D_{4\}} \psi \\
O_{A_{2}}^{\prime}=\bar{\psi} \gamma_{4} \gamma_{5} D_{4} \psi-\frac{1}{3} \sum_{i=1}^{3} \bar{\psi} \gamma_{i} \gamma_{5} D_{i} \psi
\end{gathered}
$$

The symmetrized, covariant derivatives $D$ are defined as: $D=\vec{D}-\overleftarrow{D}$, with:

$$
\vec{D}_{\mu} \psi(x)=\frac{1}{2 a}\left[U(x, \mu) \psi(x+a \hat{\mu})-U^{\dagger}(x-a \hat{\mu}, \mu) \psi(x-a \hat{\mu})\right]
$$

and similarly for the left derivative $\overleftarrow{D}$

Our calculations provide a cross check and an extension of results appearing in Refs. [3, 4, 5. Work is in progress using operators measuring the second moment of quark momentum and helicity distributions.

We have performed our calculations for a rather wide selection of values for the parameters entering the gluon and fermion actions, corresponding to present and future simulations. In particular, our results scan the whole range of admissible values for the overlap parameter. Results for different choices of parameter values can be provided by the authors upon request.

A synopsis of some of our results can be found in Ref. [6]. 


\section{Calculational setup.}

We denote the lattice action by

$$
S=S_{G}+S_{F}
$$

where $S_{G}$ is the gluon action, and $S_{F}$ is the fermion action. The gluon action we consider is written in standard notation:

$$
\begin{aligned}
S_{G}=-\frac{1}{g^{2}} & \left(c_{0} \sum_{\text {plaquette }} \operatorname{Tr} U_{p l}+c_{1} \sum_{\text {rectangle }} \operatorname{Tr} U_{r t g}\right. \\
& \left.+c_{2} \sum_{\text {chair }} \operatorname{Tr} U_{c h r}+c_{3} \sum_{\text {parallelogram }} \operatorname{Tr} U_{\text {plg }}\right)
\end{aligned}
$$

where $U_{p l}$ is the standard plaquette, while the remaining U's cover all possible closed loops containing up to six links, as indicated in Fig. 1.

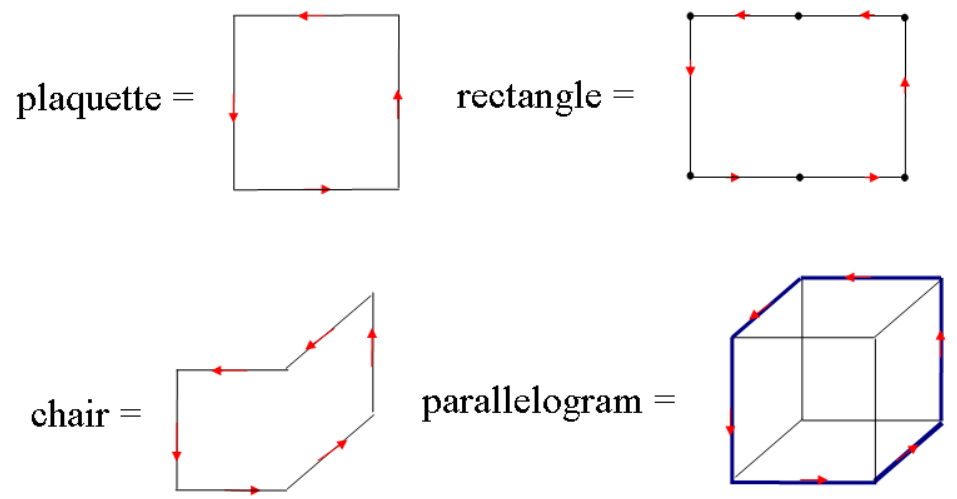

Figure 1: Closed loops corresponding to the four terms in the gluon action (12).

The coefficients $c_{0}, c_{1}, c_{2}, c_{3}$ satisfy the normalization condition:

$$
c_{0}+8 c_{1}+16 c_{2}+8 c_{3}=1
$$

As in Ref. [4], we have used the values of $c_{0}, c_{1}, c_{2}, c_{3}$, shown in Table 1 , where "Plaquette" is the standard Wilson action for gluons, "Symanzik" is the tree-level improved action [7] and TILW is the tadpole improved Luescher-Weisz action [8, 9]. Two further choices which we have considered for $c_{i}$ are the Iwasaki [10] and DBW2 [11] actions. It is worth noting that, at the 1-loop level, these coefficients enter the calculation only through the propagator and not through any vertices; given that the propagator does not depend separately on $c_{2}$ and $c_{3}$, but only on their sum, we have considered only parameter values with $c_{2}=0$.

The action for massless overlap fermions is given by [12]

$$
S_{F}=\sum_{f} \sum_{x, y} \bar{\psi}_{x}^{f} D_{N}(x, y) \psi_{y}^{f}
$$

with

$$
D_{N}=\rho\left[1+X\left(X^{\dagger} X\right)^{-\frac{1}{2}}\right]
$$




\begin{tabular}{|c|l|l|l|l|}
\multicolumn{1}{c}{ Action } & \multicolumn{1}{c}{$c_{0}$} & \multicolumn{1}{c}{$c_{1}$} & \multicolumn{1}{c}{$c_{2}$} & \multicolumn{1}{c|}{$c_{3}$} \\
\hline Plaquette & 1.0 & 0.0 & 0.0 & 0.0 \\
Symanzik & 1.66666 & -0.083333 & 0.0 & 0.0 \\
TILW1 $\beta=8.60$ & 2.31681 & -0.151791 & 0.0 & -00128098 \\
TILW2 $\beta=8.45$ & 2.34602 & -0.154846 & 0.0 & -0.0134070 \\
TILW3 $\beta=8.30$ & 2.38698 & -0.159128 & 0.0 & -0.0142442 \\
TILW4 $\beta=8.20$ & 2.41278 & -0.161827 & 0.0 & -0.0147710 \\
TILW5 $\beta=8.10$ & 2.44654 & -0.165353 & 0.0 & -0.0154645 \\
TILW6 $\beta=8.00$ & 2.48917 & -0.169805 & 0.0 & -0.0163414 \\
Iwasaki & 3.648 & -0.331 & 0.0 & 0.0 \\
DBW2 & 12.2688 & -1.4086 & 0.0 & 0.0 \\
\hline
\end{tabular}

Table 1: The values of coefficients $c_{0}, c_{1}, c_{2}, c_{3}$.

and: $X=D_{w}-\rho$. Here $D_{w}$ is the massless Wilson-Dirac operator with $r=1$, and $\rho$ is a free parameter whose value must be in the range $0<\rho<2$ in order to guarantee the correct pole structure of $D_{N}$.

\section{Results.}

\section{Quark propagator}

Let us consider the massless quark propagator $S_{N}$ first. In momentum space, the inverse of $S_{N}$ can be written as:

$$
S_{N}^{-1}=i \gamma_{\mu} p_{\mu}\left(1-\frac{g^{2} C_{F}}{16 \pi^{2}} \Sigma_{1}\right)
$$

with $C_{F}=\left(N^{2}-1\right) / 2 N$, and $\Sigma_{1}(a, p)=\log \left(a^{2} p^{2}\right)+b_{\Sigma}$ (Feynman gauge). In Eq. (16), the factor in parentheses is the quark wave function renormalization; in the MOM scheme we have:

$$
Z_{\psi}^{M O M}(a, \mu)=1-\frac{g^{2} C_{F}}{16 \pi^{2}} \Sigma_{1}(a, \mu)
$$

The diagrams that contribute to one loop order are shown in Fig. 2. Our results for $b_{\Sigma}$ are shown in Fig. 3 and, on a different scale, in Fig. 4. One observes a divergence of this quantity for $\rho \rightarrow 0$, which can be expected, since the fermion propagator behaves singularly in this limit. Numerical values of $b_{\Sigma}$ are listed in Table 2 for typical values of $\rho: \rho=0.6,1.0,1.4$. 

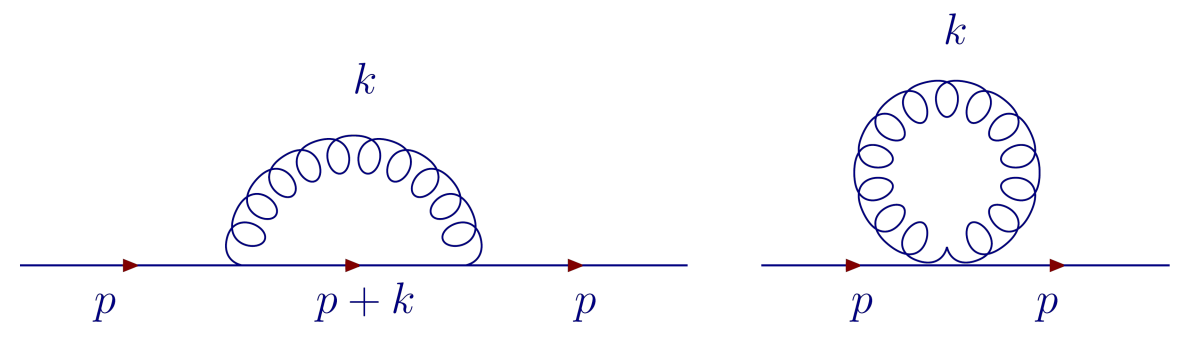

Figure 2: One-loop diagrams contributing to the quark self-energy.

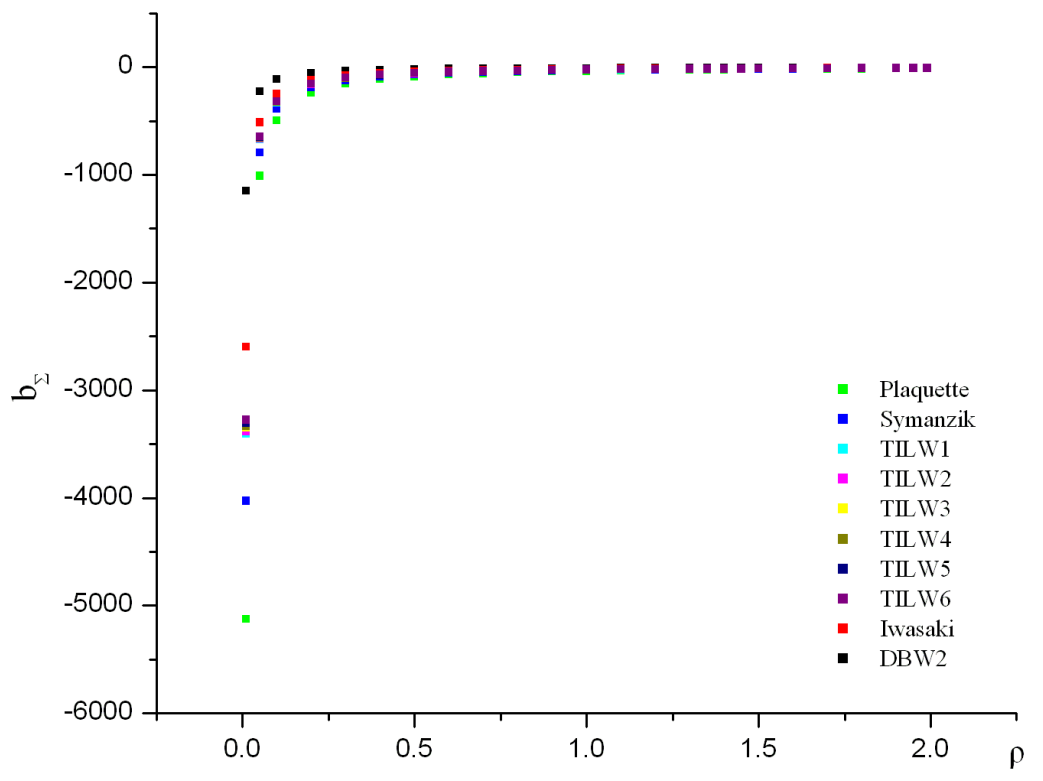

Figure 3: $b_{\Sigma}$ as a function of the overlap parameter $\rho$, for different gluon actions. 


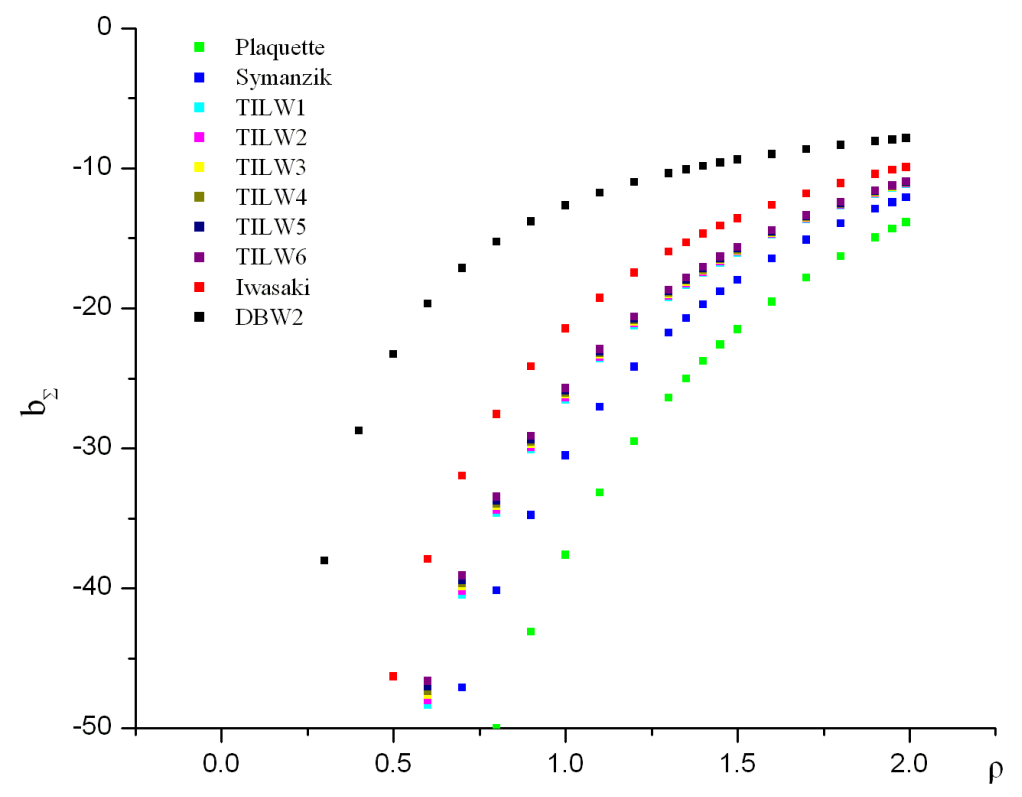

Figure 4: $b_{\Sigma}$ as a function of the overlap parameter $\rho$, shown on a different scale.

\section{Quark bilinears}

Let us consider local operators of the form

$$
O_{X}=\bar{\psi}(x) \Gamma^{X} \psi(x)
$$

where $X=S, P, V, A, T$, and $\Gamma^{S}=1, \Gamma^{P}=\gamma_{5}, \Gamma^{V}=\gamma_{\mu}, \Gamma^{A}=\gamma_{\mu} \gamma_{5}, \Gamma^{T}=\sigma_{\mu \nu} \gamma_{5}$. We denote the amputated Green's function of the operator $O_{X}$ by $\Lambda^{X}$. The final results are (in Feynman gauge):

$$
\begin{gathered}
\Lambda^{S, P}=\left\{1, \gamma_{5}\right\}+\frac{g^{2} C_{F}}{16 \pi^{2}}\left[-4 \log \left(a^{2} p^{2}\right)+b_{S, P}\right]\left\{1, \gamma_{5}\right\} \\
\Lambda_{\mu}^{V, A}=\left\{\gamma_{\mu}, \gamma_{\mu} \gamma_{5}\right\}+\frac{g^{2} C_{F}}{16 \pi^{2}}\left[-\gamma_{\mu}\left(\log \left(a^{2} p^{2}\right)+b_{V, A}\right)+2 \frac{p_{\mu} \not p}{p^{2}}\right]\left\{1, \gamma_{5}\right\} \\
\Lambda_{\mu, \nu}^{T}=\sigma_{\mu, \nu} \gamma_{5}+\frac{g^{2} C_{F}}{16 \pi^{2}} b_{T} \sigma_{\mu, \nu} \gamma_{5}
\end{gathered}
$$

Where $b_{S}=b_{P}$ and $b_{V}=b_{A}$. Among the diagrams appearing in Fig. 5, only the first one (Vertex) contributes to $\Lambda^{X}$. 

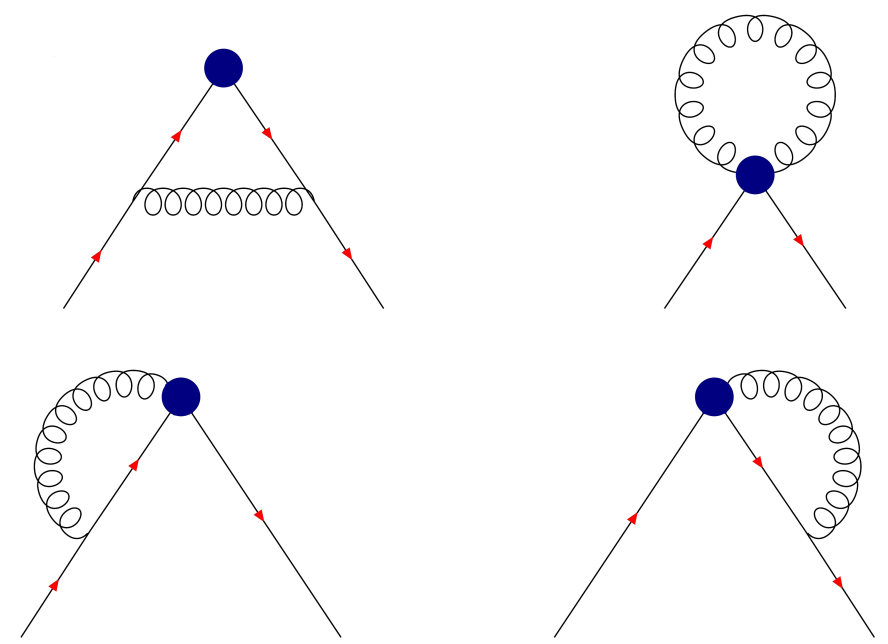

Figure 5: One-loop lattice Feynman diagrams contributing to the amputated Green's functions of $O_{X}$ and $O_{\mu \mu_{1} \ldots \mu_{n}}$. From left to right: vertex, operator tadpole, left sail and right sail.

Our results provide a cross check and an extension of results appearing in Refs. 3 , 4. They are shown in Figures $3+4\left(b_{\Sigma}\right), 6\left(b_{S, P}\right), 7\left(b_{V, A}\right), 8\left(b_{T}\right)$ for typical values of the overlap parameter $\rho$. The same quantities are also listed in Tables $2,3,4,5$, respectively, for the representative choices: $\rho=0.6,1.0,1.4$. In all cases which can be compared, our results agree with those of Refs. [3, 4]. There is one exception, regarding the values of $b_{T}$ for cases other than the standard plaquette $b_{T}^{\text {plaq }}$ : In these cases, our results for $b_{T}-b_{T}^{\text {plaq }}$ have the opposite sign compared to Ref. [4].

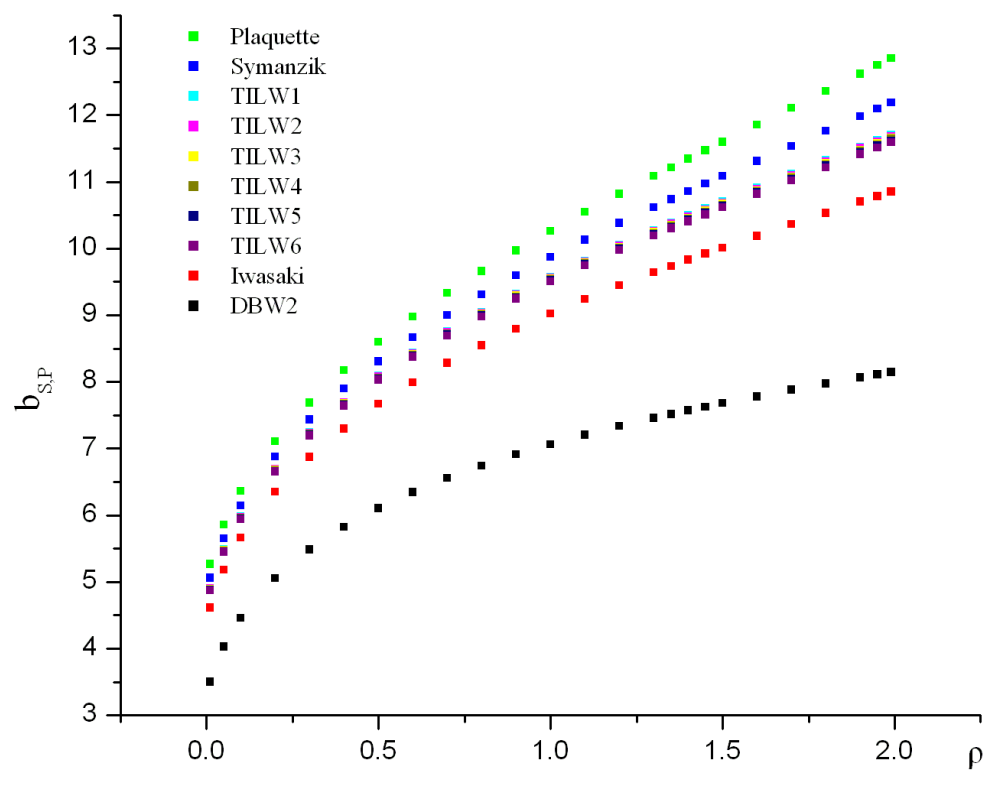

Figure $6: b_{S, P}$ as a function of the overlap parameter $\rho$, for different gluon actions. 


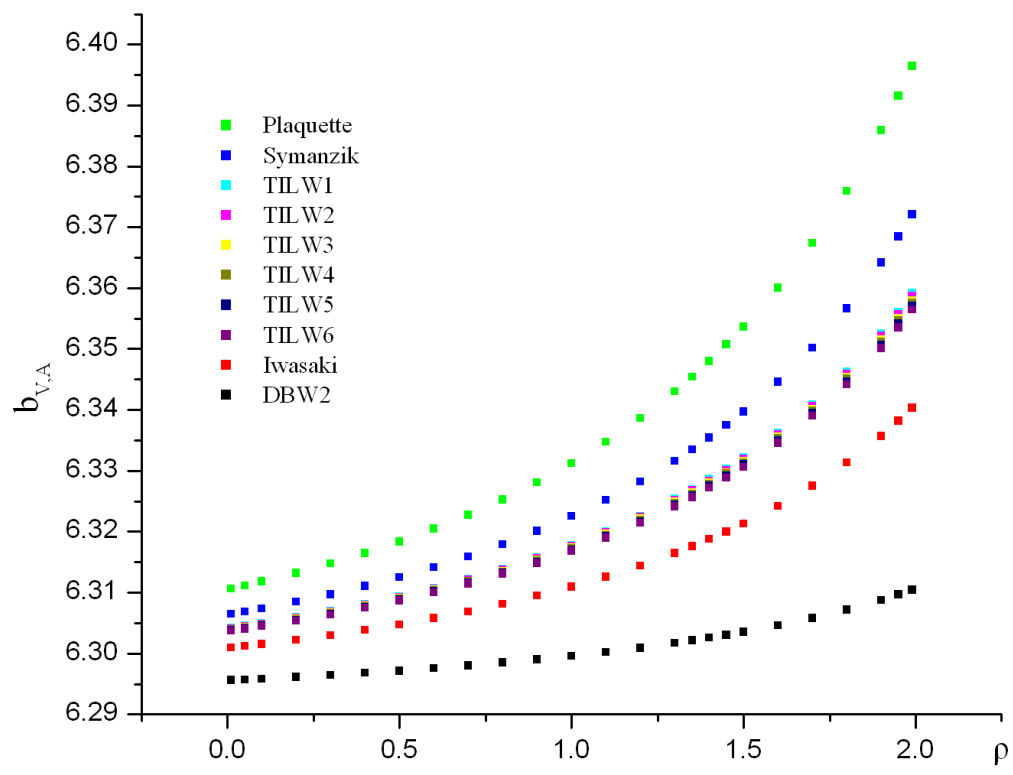

Figure 7: $b_{V, A}$ as a function of the overlap parameter $\rho$, for different gluon actions.

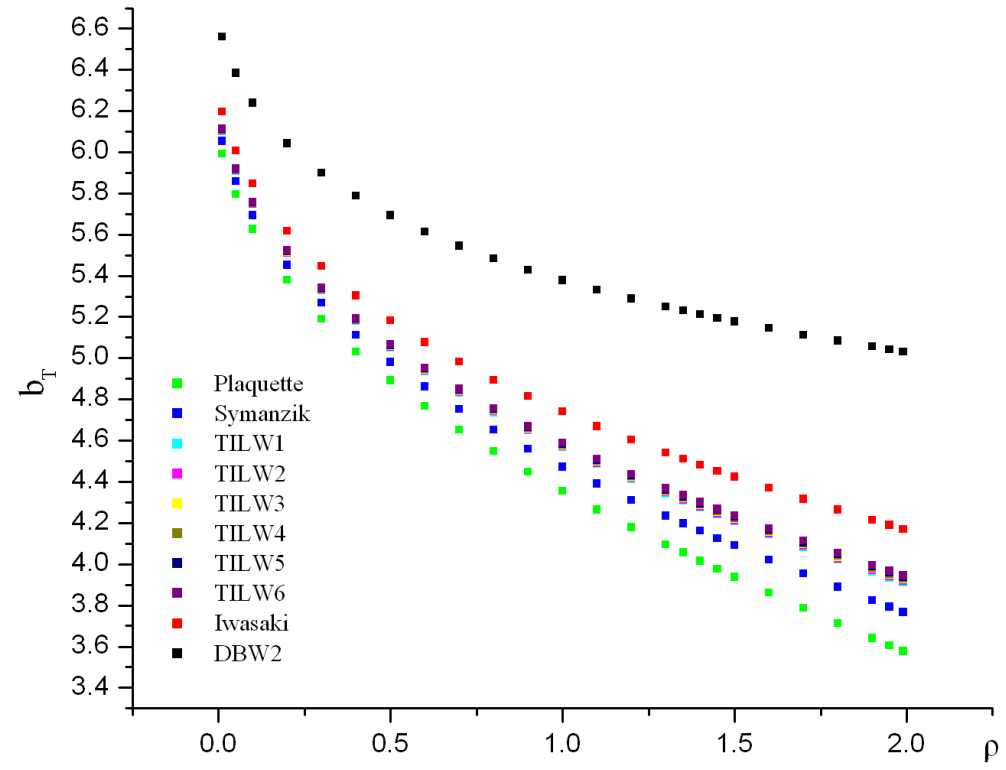

Figure 8: $b_{T}$ as a function of the overlap parameter $\rho$, for different gluon actions. 


\section{First moment of quark distributions.}

We have calculated the 1-loop renormalization coefficients of the operators $O_{V_{2}}, O_{V_{2}}^{\prime}$ which are the symmetric off-diagonal, and the traceless diagonal parts, respectively, of the operator:

$$
O_{\mu, \nu}=\bar{\psi} \gamma_{\{\mu} D_{\nu\}} \psi
$$

The axial counterparts $O_{A_{2}}, O_{A_{2}}^{\prime}$ of the above operators renormalize in the same way. Using the notation of Ref. [5], we find for the amputated Green's function of $O_{\mu, \nu}$ in the Feynman gauge:

$$
\Lambda_{\mu, \nu}(a, p)=\gamma_{\{\mu} p_{\nu\}}+\frac{g^{2} C_{F}}{16 \pi^{2}}\left[\left(\frac{5}{3} \log \left(a^{2} p^{2}\right)+b\right) \gamma_{\{\mu} p_{\nu\}}+b^{\prime} \delta_{\mu \nu} \gamma_{\nu} p_{\nu}-\frac{4}{3} \frac{p_{\mu} p_{\nu}}{p^{2}} \not p\right]
$$

The quantities $b, b^{\prime}$ correspond to $\left(b_{1}+b_{2}\right), b_{4}$ of Ref. [5], respectively. All diagrams of Fig. 5 contribute in this case. The rational coefficients $5 / 3$ and $-4 / 3$ in Eq. (23) check with those of Ref. [5]. The values of $b$ and $b^{\prime}$ are shown in Figures 9 and 10; they are in agreement with the quantities $b_{1}+b_{2}$ and $b_{4}$, respectively, as defined in Ref. [5], for the choice of parameters considered in that reference. In Tables 6 and 7 we list our results for $b$ and $b^{\prime}$, respectively, with the choice of values for $\rho: \rho=0.6,1.0,1.4$.

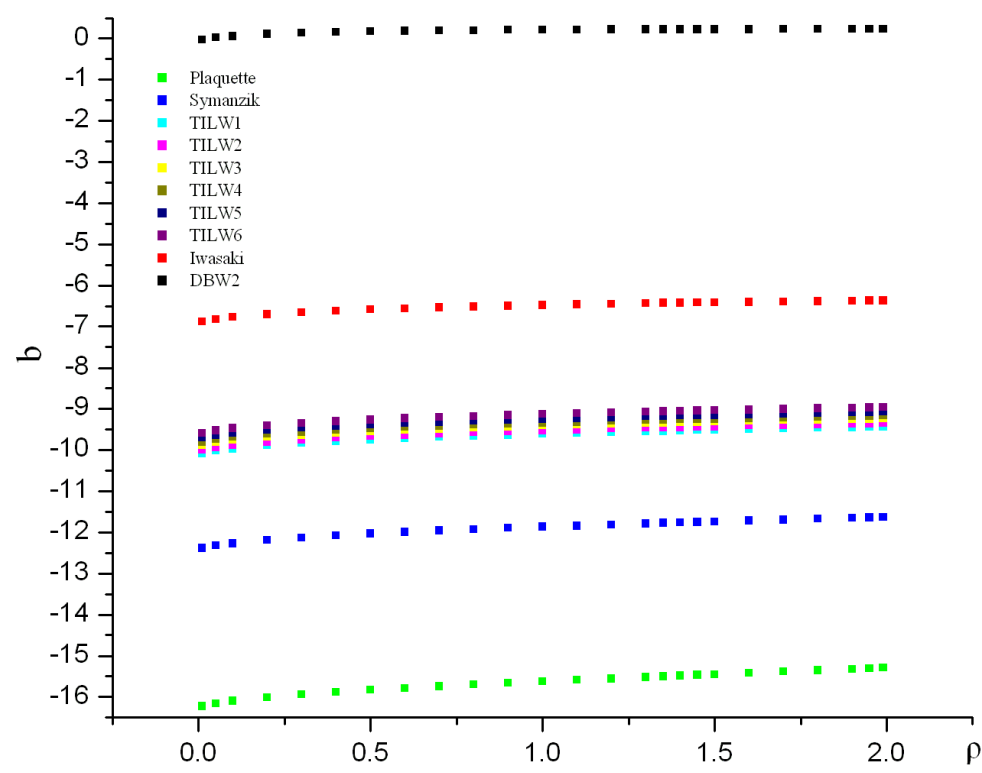

Figure 9: $b$ as a function of the overlap parameter $\rho$, for different gluon actions. 


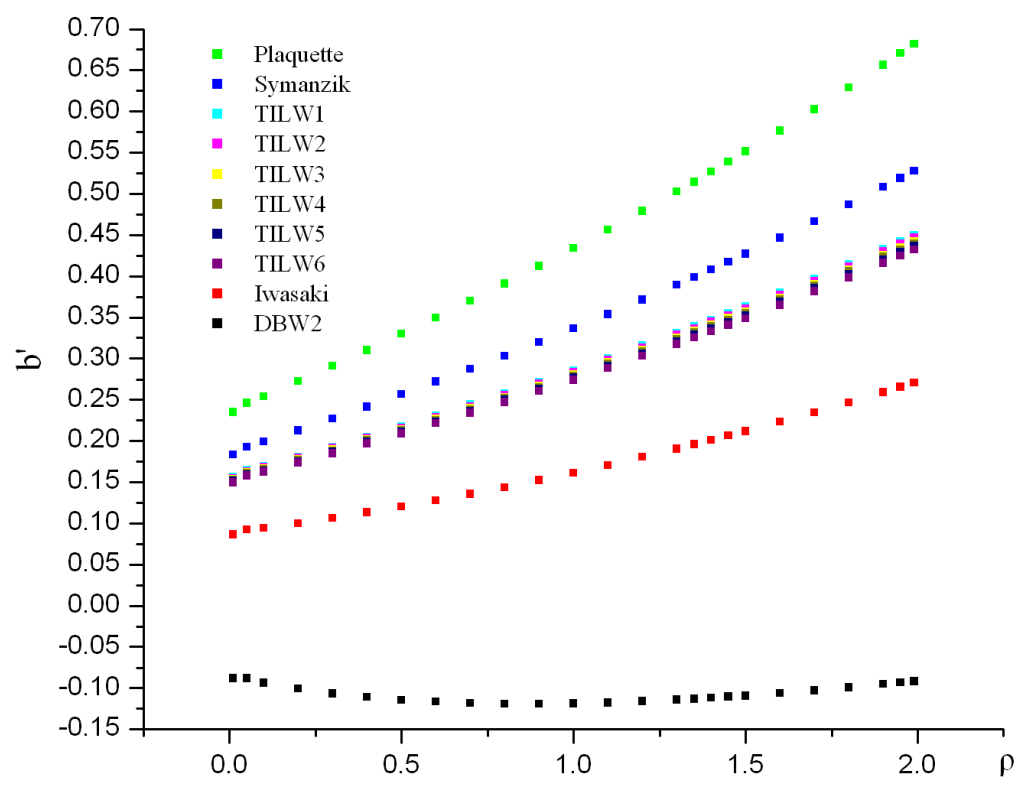

Figure 10: $b^{\prime}$ as a function of the overlap parameter $\rho$, for different gluon actions.

All 1-loop calculations presented in this work were performed using our Mathematica package for lattice perturbation theory. Numerically inverting the gluon propagator turned out to be less CPU consuming, as compared to using an analytic expression for the inverse. We used a rather wide spectrum of values for the Symanzik parameters $c_{i}$; given that the dependence of 1-loop results on $c_{2}$ and $c_{3}$ is only through their sum $c_{2}+c_{3}$, we have chosen $c_{2}=0$ without loss of generality. For the overlap parameter $\rho$ we have explored in detail its full spectrum of admissible values. Results for further values of the parameters can be provided by the authors upon request.

We observe a rather smooth dependence of our results on $\rho$, and this fact allows for a safe interpolation to any additional $\rho$-values in the allowed range $0<\rho<2$. A possible exception is the behaviour of $b_{\Sigma}$ near the endpoint $\rho \rightarrow 0$, which can be expected given the incorrect pole structure of the fermion operator in that limit. The dependence on $\rho$ is even less pronounced for the DBW2 action.

Results for the various choices of parameters for the Tadpole Improved Lüscher-Weisz (TILW) action are in many cases almost indistinguishable from one another, due to the proximity in these parameter values; consequently, it is straightforward to interpolate accurately our results to other sets of values which were not considered in this work.

It is worth noticing that both $b$ and $b^{\prime}$ are noticeably suppressed in the case of the DBW2 action. A similar effect has been observed in the perturbative renormalization of both the topological charge and susceptibility, using this action [13. This fact is an indication in favour of this action for purposes of numerical simulation.

Some related quantities of interest for the study of structure functions are the second 
moments of quark momentum and helicity distributions, as well as non-forward matrix elements of various fermion bilinears. We expect to address these issues in a future report.

\section{References}

[1] S. Capitani, Perturbative renormalization of the first two moments of non-singlet quark distributions with overlap fermions, Nucl.Phys. B592 (2001) 183.

[2] T. DeGrand, A. Hasenfratz, T. Kovacs, Improving the chiral properties of lattice fermions, Phys. Rev. D67 (2003) 054501.

[3] C. Alexandrou, E. Follana, H. Panagopoulos and E. Vicari, One-loop renormalization of fermionic currents with the overlap-Dirac operator, Nucl. Phys. B580 (2000) 394.

[4] R. Horsley, H. Perlt, P. E. L. Rakow, G. Schierholz and A. Schiller, One-loop renormalization of quark bilinears for overlap fermions with improved gauge actions, Nucl. Phys. B693 (2004) 3.

[5] R. Horsley, H. Perlt, P. E. L. Rakow, G. Schierholz and A. Schiller, Renormalization of one-link quark operators for overlap fermions with Lüscher-Weisz gauge action, Phys. Lett. B628 (2005) 66.

[6] M.Ioannou and H.Panagopoulos, Perturbative renormalization in parton distribution functions using improved actions, PoS (LAT2005) 229.

[7] K. Symanzik, Continuum limit and improved action in lattice theories. 1. Principles and $p h i^{* *} 4$ theory, Nucl. Phys. B226 (1983) 187.

[8] M. Lüscher and P. Weisz, On-shell improved lattice gauge theories, Commun. Math. Phys. 97 (1985) 59 [Erratum-ibid. 98 (1985) 433].

[9] M. G. Alford, W. Dimm, G. P. Lepage, G. Hockney and P. B. Mackenzie, QCD on small computers, Phys. Lett. B361 (1995) 87.

[10] Y. Iwasaki, Renormalization group analysis of lattice theories and improved lattice action. 2. Four-dimensional nonabelian SU(N) gauge model, UTHEP-118 (1983).

[11] T. Takaishi, Heavy quark potential and effective actions on blocked configurations, Phys. Rev. D54 (1996) 1050.

[12] H. Neuberger, Exactly massless quarks on the lattice, Phys. Lett. B417 (1998) 141; More about exactly massless quarks on the lattice, Phys. Lett. B427 (1998) 353.

[13] A. Skouroupathis and H. Panagopoulos, Additive and multiplicative renormalization of topological charge with improved gluon/fermion actions: A test case for 3-loop vacuum calculations, using overlap or clover fermions, Phys. Rev. D72 (2005) 094509. 


\section{TABLES}

\begin{tabular}{|c|l|l|l|}
\multicolumn{1}{c}{ Action } & \multicolumn{1}{c}{$\rho=0.6$} & \multicolumn{1}{c}{$\rho=1.0$} & $\rho=1.4$ \\
\hline Plaquette & $-70.8442846023(6)$ & $-37.6306267984(3)$ & $-23.7659791950(4)$ \\
Symanzik & $-56.4356742205(6)$ & $-30.5049192399(3)$ & $-19.7319952945(2)$ \\
TILW1 $\beta=8.60$ & $-48.315432542(1)$ & $-26.5273492659(2)$ & $-17.5090552592(4)$ \\
TILW2 $\beta=8.45$ & $-48.020432621(1)$ & $-26.3832716142(5)$ & $-17.4287897913(1)$ \\
TILW3 $\beta=8.30$ & $-47.614837428(1)$ & $-26.1852273116(3)$ & $-17.3184864381(3)$ \\
TILW4 $\beta=8.20$ & $-47.363843382(1)$ & $-26.0626992727(4)$ & $-17.2502579516(4)$ \\
TILW5 $\beta=8.10$ & $-47.040843009(1)$ & $-25.9050530413(4)$ & $-17.1624938296(4)$ \\
TILW6 $\beta=8.00$ & $-46.641145138(1)$ & $-25.7100225495(5)$ & $-17.0539447038(2)$ \\
Iwasaki & $-37.911348699(2)$ & $-21.454735885(1)$ & $-14.680753624(5)$ \\
DBW2 & $-19.673287561(6)$ & $-12.675353551(3)$ & $-9.840831462(1)$ \\
\hline
\end{tabular}

Table 2: The contribution $b_{\Sigma}$ to the self energy for various actions.

Action

Plaquette Symanzik

TILW1 $\beta=8.60$

TILW2 $\beta=8.45$

TILW3 $\beta=8.30$

TILW4 $\beta=8.20$

TILW5 $\beta=8.10$

TILW6 $\beta=8.00$

Iwasaki

DBW2

$$
\rho=0.6
$$

\begin{tabular}{|l|}
$8.9798281638(5)$ \\
$8.6712333358(5)$ \\
$8.4347849739(6)$ \\
$8.4249104780(5)$ \\
$8.4111618345(5)$ \\
$8.4025516764(5)$ \\
$8.3913573861(6)$ \\
$8.3773207579(2)$ \\
$7.989846185(1)$ \\
$6.346306461(1)$
\end{tabular}

$6.346306461(1)$

$$
\rho=1.0
$$

$\rho=1.4$

\begin{tabular}{|c|l|}
\hline $10.26190085663(6)$ & $11.34335740638(2)$ \\
$9.87030941138(2)$ & $10.85599048485(3)$ \\
$9.57373721242(1)$ & $10.4927473931(1)$ \\
$9.56141189336(1)$ & $10.4777493701(1)$ \\
$9.54425880565(2)$ & $10.45688939118(1)$ \\
$9.53352128912(2)$ & $10.44383891932(1)$ \\
$9.51956660551(1)$ & $10.4268869200(1)$ \\
$9.50207732589(2)$ & $10.4056548274(1)$ \\
$9.02297610282(2)$ & $9.82968352735(1)$ \\
$7.0639464328(2)$ & $7.571097190(2)$ \\
\hline
\end{tabular}

Table 3: The contribution $b_{S, P}$ to $\Lambda^{S, P}$ for various actions.

Action

Plaquette

Symanzik

TILW1 $\beta=8.60$

TILW2 $\beta=8.45$

TILW3 $\beta=8.30$

TILW4 $\beta=8.20$

TILW5 $\beta=8.10$

TILW6 $\beta=8.00$

Iwasaki

DBW2 $\rho=0.6$

\begin{tabular}{|l|l|l|}
$6.3204575633(1)$ & $6.33125072110(1)$ & $6.34801586915(1)$ \\
$6.3141428350(1)$ & $6.32252999501(1)$ & $6.3354222684(1)$ \\
$6.3107172748(1)$ & $6.31780175061(1)$ & $6.32862040074(3)$ \\
$6.3105909672(1)$ & $6.31762724546(3)$ & $6.32836942659(2)$ \\
$6.3104170577(1)$ & $6.31738695411(2)$ & $6.32802384701(4)$ \\
$6.3103092794(1)$ & $6.31723802434(3)$ & $6.32780966499(3)$ \\
$6.3101704948(1)$ & $6.31704624086(2)$ & $6.32753386701(3)$ \\
$6.3099985210(1)$ & $6.31680857518(1)$ & $6.32719209974(1)$ \\
$6.3057833598(1)$ & $6.31095730979(1)$ & $6.31875098630(4)$ \\
$6.2975701799(1)$ & $6.2996002928(1)$ & $6.30255799548(6)$ \\
\hline
\end{tabular}

Table 4: The contribution $b_{V, A}$ to $\Lambda^{V, A}$ for various actions. 
Action $\rho=0.6$ $\rho=1.0$ $\rho=1.4$

\begin{tabular}{|c|l|l|l|}
\hline Plaquette & $4.7673340248(1)$ & $4.35436733681(1)$ & $4.0162353498(7)$ \\
Symanzik & $4.86177933052(5)$ & $4.4732701854(1)$ & $4.16189952383(6)$ \\
TILW1 $\beta=8.60$ & $4.9360280383(1)$ & $4.56582325908(4)$ & $4.27391139850(6)$ \\
TILW2 $\beta=8.45$ & $4.93915112696(5)$ & $4.56969902605(5)$ & $4.27857610740(2)$ \\
TILW3 $\beta=8.30$ & $4.94350212841(2)$ & $4.57509633367(4)$ & $4.28506866099(4)$ \\
TILW4 $\beta=8.20$ & $4.94622847715(4)$ & $4.57847693279(6)$ & $4.28913324224(3)$ \\
TILW5 $\beta=8.10$ & $4.9497748610(1)$ & $4.58287278284(4)$ & $4.29441617825(3)$ \\
TILW6 $\beta=8.00$ & $4.95422443865(1)$ & $4.58838565488(6)$ & $4.30103785269(2)$ \\
Iwasaki & $5.0777624156(2)$ & $4.7402843779(1)$ & $4.48177346976(3)$ \\
DBW2 & $5.6146580874(6)$ & $5.378151584(1)$ & $5.213044934(1)$ \\
\hline
\end{tabular}

Table 5: The contribution $b_{T}$ to $\Lambda^{T}$ for various actions.

Action $\rho=0.6$ $\rho=1.0$ $\rho=1.4$

\begin{tabular}{|c|r|r|r|}
\hline Plaquette & $-15.7863271766(1)$ & $-15.6250817532(1)$ & $-15.4858619898(1)$ \\
Symanzik & $-11.9937499031(2)$ & $-11.8664388808(2)$ & $-11.7640746811(2)$ \\
TILW1 $\beta=8.60$ & $-9.7160722504(2)$ & $-9.6095867774(2)$ & $-9.5287008568(2)$ \\
TILW2 $\beta=8.45$ & $-9.6309328038(2)$ & $-9.5252267216(1)$ & $-9.4451255929(1)$ \\
TILW3 $\beta=8.30$ & $-9.5135691580(2)$ & $-9.4089372737(1)$ & $-9.3299153795(1)$ \\
TILW4 $\beta=8.20$ & $-9.4407612246(2)$ & $-9.3367955747(1)$ & $-9.2584416246(1)$ \\
TILW5 $\beta=8.10$ & $-9.3468642074(4)$ & $-9.2437578051(4)$ & $-9.1662637020(4)$ \\
TILW6 $\beta=8.00$ & $-9.2303490445(1)$ & $-9.1283086414(1)$ & $-9.0518787415(1)$ \\
Iwasaki & $-6.5572678902(2)$ & $-6.4778880567(1)$ & $-6.4232808744(1)$ \\
DBW2 & $0.185440914(4)$ & $0.211859722(4)$ & $0.222852646(3)$ \\
\hline
\end{tabular}

Table 6: The contribution $b$ to $\Lambda_{\mu \nu}$ for various actions.

Action $\rho=0.6$ $\rho=1.0$ $\rho=1.4$

\begin{tabular}{|c|c|c|c|}
\hline Plaquette & $0.3497088259(1)$ & $0.4340050439(1)$ & $0.5267146951(2)$ \\
Symanzik & $0.2719708888(4)$ & $0.3367316099(2)$ & $0.4080895543(1)$ \\
TILW1 $\beta=8.60$ & $0.2307195514(3)$ & $0.2858316439(2)$ & $0.3469216320(2)$ \\
TILW2 $\beta=8.45$ & $0.2290786112(2)$ & $0.2838197180(1)$ & $0.3445199656(2)$ \\
TILW3 $\beta=8.30$ & $0.2268014376(4)$ & $0.2810293893(1)$ & $0.3411911844(3)$ \\
TILW4 $\beta=8.20$ & $0.2253789601(2)$ & $0.2792873534(1)$ & $0.3391142249(3)$ \\
TILW5 $\beta=8.10$ & $0.2235391956(1)$ & $0.2770353882(1)$ & $0.3364306054(3)$ \\
TILW6 $\beta=8.00$ & $0.2212413920(1)$ & $0.2742245511(1)$ & $0.3330831698(2)$ \\
Iwasaki & $0.1277382408(3)$ & $0.1614096997(1)$ & $0.2010432525(2)$ \\
DBW2 & $-0.116651374(1)$ & $-0.118539399(1)$ & $-0.111845323(1)$ \\
\hline
\end{tabular}

Table 7: The contribution $b^{\prime}$ to $\Lambda_{\mu \nu}$ for various actions. 